\title{
Stopping power: Effect of the projectile deceleration
}

\author{
Roman Kompaneets * Alexei V. Ivlev, and Gregor E. Morfill \\ Max-Planck-Institut für extraterrestrische Physik, Giessenbachstr. 1, 85748 Garching, Germany
}

(Dated: April 28, 2022)

\begin{abstract}
The stopping force is the force exerted on a charged projectile by the excess charge of the wake generated by the projectile in the surrounding plasma. Since the wake does not instantly adjust to the projectile velocity, the stopping force should be affected by the projectile deceleration caused by the stopping force itself. We address this effect by deriving the corresponding correction to the stopping force in a cold plasma. We find that if the projectile is an ion, the correction is small when the stopping force is due to the plasma electrons, but can be significant when the stopping force is due to the ions.
\end{abstract}

PACS numbers: 52.40.Mj

\section{INTRODUCTION}

The calculation of the stopping force, which is the force exerted by a moving charged projectile on itself through the perturbation of the surrounding plasma, is a classic problem, with applications spanning from fusion [1-3] to dusty plasmas [4 8]. In the linear perturbation approximation, the calculation of the stopping force is straightforward once the dielectric function is specified and the projectile velocity is assumed to be constant [1, 9]; for a cold plasma, it results in the Bohr stopping force [1, 10].

While the standard calculation assumes a constant projectile velocity, in reality projectiles experience deceleration for the stopping force itself. Since the plasma perturbation, or wake, does not instantly adjust to the projectile velocity, the momentary stopping force should be different from the one predicted by the standard calculation using the momentary value of the velocity.

This difference, that is, the effect of the deceleration on the stopping force, is not obvious to estimate. For a cold plasma and uniform motion, as well known, one must include a damping rate to avoid a singularity of the integral for the stopping force [11, 12]; the inclusion of a deceleration, as we will show, results in a competition between the deceleration and the damping [Eq. (14)]. When the deceleration dominates, which is a quite common regime [as shown in Sec. III], it is difficult to make simple estimates on the effect of the deceleration on the stopping force without making accurate calculations.

Given the universality of the effect and that its magnitude is not obvious, it needs to be rigorously addressed, which is the object of this paper.

*Electronic address: kompaneets@mpe.mpg.de

\section{THEORY}

Let us consider a point charge $q$ moving through a plasma according to

$$
x(t)=y(t)=0, \quad z(t)=\frac{a t^{2}}{2}
$$

with a constant $a>0$ starting from $t=-\infty$ and derive the stopping force on that charge at any $t<0$, the decelerating phase of the motion, first assuming an arbitrary dielectric function $D(\omega, \mathbf{k})$ and then focusing on the case of a cold plasma. Note that by making the assumption of a constant deceleration, we probe into the principal effect of non-uniform motion and neglect the higher-order corrections.

For arbitrary $D(\omega, \mathbf{k})$, the steps to derive an integral expression for the stopping force are rather straightforward. The extraneous charge density in our case is

$$
\rho(\mathbf{r}, t)=q \delta(x) \delta(y) \delta\left(z-\frac{a t^{2}}{2}\right),
$$

so its Fourier transform in space and time is

$$
\begin{aligned}
\hat{\rho}(\omega, \mathbf{k}) & =\int_{-\infty}^{\infty} d t \int d \mathbf{r} \rho(\mathbf{r}, t) \exp (i \omega t-i \mathbf{k} \cdot \mathbf{r}) \\
& =q \sqrt{\frac{\pi}{\left|k_{z}\right| a}} \exp \left(\frac{i \omega^{2}}{2 k_{z} a}\right)\left(1-\frac{i k_{z}}{\left|k_{z}\right|}\right) .
\end{aligned}
$$

We use the linear perturbation approximation,

$$
\hat{\varphi}_{\text {diff }}(\omega, \mathbf{k})=\frac{\hat{\rho}(\omega, \mathbf{k})}{\varepsilon_{0} k^{2}}\left(\frac{1}{D(\omega, \mathbf{k})}-1\right),
$$

where $\hat{\varphi}_{\text {diff }}(\omega, \mathbf{k})$ is the Fourier transform in space and time of the difference $\varphi_{\text {diff }}(\mathbf{r}, t)$ between the potential induced by the charge $q$ and its Coulomb potential, and $\varepsilon_{0}$ is the permittivity of free space. The stopping force

$$
F(t)=-q \frac{\partial \varphi_{\mathrm{diff}}(\mathbf{r}, t)}{\partial z},
$$

where the derivative is taken at the charge location, is found by performing the inverse Fourier transform of 
Eq. (4). We simplify the resulting expression by (i) confining the integration to positive $k_{z}$ - for this we use the general symmetry properties $\operatorname{Re} D(\omega, \mathbf{k})=\operatorname{Re} D(-\omega,-\mathbf{k})$ and $\operatorname{Im} D(\omega, \mathbf{k})=-\operatorname{Im} D(-\omega,-\mathbf{k})$ - and (ii) making the substitution

$$
\omega=\eta \sqrt{k_{z} a}+k_{z} a t,
$$

where $\eta$ is a new integration variable. The result is

$$
\begin{array}{r}
F=-\frac{q^{2} \sqrt{\pi}}{8 \pi^{4} \varepsilon_{0}} \operatorname{Re} \int_{k_{z}>0} \frac{k_{z} d \mathbf{k}}{k^{2}} \int_{-\infty}^{\infty} d \eta(1+i) \exp \left(\frac{i \eta^{2}}{2}\right) \\
\times\left[\frac{1}{D\left(k_{z} v_{z}+\eta \sqrt{k_{z} a}, \mathbf{k}\right)}-1\right]
\end{array}
$$

where $v_{z}=a t$ is the momentary $z$-velocity of the charge. Here and in the following, the integration over $\mathbf{k}$ is restricted to $\left|k_{z}\right|<k_{\max }$ and $k_{\perp}\left(=\sqrt{k_{x}^{2}+k_{y}^{2}}\right)<k_{\max }$, where $k_{\max }$ is the inverse length at which the nonlinear effects become significant, in order to avoid the well-known logarithmic divergence at large wave numbers [1, 9]. (Note that we could alternatively restrict the integration by $k<k_{\max }$ as in Ref. [1]; both approaches are valid when $k_{\max }$ is sufficiently large for the linear perturbation approximation to be applicable.)

It is easy to see that for $a=0 \mathrm{Eq}$. (7) reduces to the well-known expression for the stopping force on a uniformly moving charge for an arbitrary dielectric function [9].

For a nonzero $a$, even if $a$ is assumed to be small, it seems impossible to substantially simplify Eq. (7) unless $D(\omega, \mathbf{k})$ is specified. For instance, the series expansion of the integrand in Eq. (77) in powers of $\sqrt{a}$ (with $v_{z}$ being an independent parameter) produces terms $\propto$ $\exp \left(i \eta^{2} / 2\right) \eta^{n} a^{n / 2}$, integrals of which over $\eta$ diverge for non-zero $n$, while methods of complex variable integration, employed below, can be used only when the analytical properties of $D(\omega, \mathbf{k})$ are known.

So let us focus on the case of a cold plasma, assuming $[12,13]$

$$
D(\omega, \mathbf{k})=1-\frac{\omega_{\mathrm{pe}}^{2}}{\left(\omega+i 0^{+}\right)^{2}},
$$

where $\omega_{\text {pe }}$ is the plasma frequency. The term $i 0^{+}$(where $0^{+}$is an infinitesimal positive number) represents the collisional and Landau damping and is important as it removes the singularity of the integral in Eq. (7) [11, 12]. We replace the term $i 0^{+}$by a finite imaginary number $i \nu$ and keep considering it finite for a while (before taking the limit $\nu \rightarrow 0$ ), which will allows us to make estimates on the role of finite damping. Note that for a cold plasma, the upper limit $k_{\max }$ is [1]

$$
k_{\max }=\frac{4 \pi \varepsilon_{0} m_{\mathrm{e}} v_{z}^{2}}{|q| e},
$$

where $m_{\mathrm{e}}$ is the electron mass and $e$ is the elementary charge.
We substitute the dielectric function (8) (with $i 0^{+}$replaced by $i \nu$ ) to Eq. (7) and normalize the quantities as follows:

$$
\begin{array}{r}
F\left(\frac{q^{2} \omega_{\mathrm{pe}}^{2}}{4 \pi \varepsilon_{0} v_{z}^{2}}\right)^{-1} \rightarrow F, \quad \frac{\mathbf{k}\left|v_{z}\right|}{\omega_{\mathrm{pe}}} \rightarrow \mathbf{k}, \\
\frac{a}{\left|v_{z}\right| \omega_{\mathrm{pe}}} \rightarrow a, \quad \frac{\nu}{\omega_{\mathrm{pe}}} \rightarrow \nu, \quad \frac{k_{\mathrm{max}}\left|v_{z}\right|}{\omega_{\mathrm{pe}}} \rightarrow k_{\max },
\end{array}
$$

which yields (for $v_{z}<0$ )

$$
\begin{array}{r}
F=-\frac{1}{2 \pi^{5 / 2}} \operatorname{Re} \int_{k_{z}>0} \frac{k_{z} d \mathbf{k}}{k^{2}} \\
\times \int_{-\infty}^{\infty} \frac{(1+i) \exp \left(i \eta^{2} / 2\right) d \eta}{\left(k_{z}-\eta \sqrt{k_{z} a}-i \nu\right)^{2}-1} .
\end{array}
$$

As noted above, the formal expansion of the integrand in powers of $\sqrt{a}$ produces divergent integrals over $\eta$, which is because of a slow convergence of the original integral over $\eta$, so we need to transform it to improve the convergence. For this purpose, we modify the contour of integration as shown in Fig. 1 - according to Cauchy's theorem, this does not change the integration result. It is easy to show that since we choose the distance from the vertical lines of the contour to the imaginary axis to be infinitely large, the contributions from them are zero. We get

$$
F=F^{\text {pole }}+F^{\text {line }},
$$

where the contribution from the pole and the line are, respectively,

$$
\begin{aligned}
F^{\text {pole }}=\frac{1}{2 \pi^{3 / 2}} \operatorname{Re} & \int_{0<k_{z}<1-\nu} \frac{d \mathbf{k}}{k^{2}}(1-i) \sqrt{\frac{k_{z}}{a}} \\
& \times \exp \left[\frac{i\left(k_{z}-1-i \nu\right)^{2}}{2 k_{z} a}\right],
\end{aligned}
$$

and

$$
\begin{aligned}
F^{\text {line }}= & -\frac{1}{\pi^{5 / 2}} \operatorname{Re} \int_{k_{z}>0} \frac{i k_{z} d \mathbf{k}}{k^{2}} \int_{-\infty}^{\infty} d \xi \\
& \times \frac{\exp \left(-\xi^{2}\right)}{\left[k_{z}-\xi(1+i) \sqrt{k_{z} a}-i \nu\right]^{2}-1} .
\end{aligned}
$$

[Here, we have made the substitution $\eta=(1+i) \xi$.] Now the convergence is considerably improved as the integral over $\xi$ converges exponentially fast.

We note that when both $a$ and $\nu$ are infinitesimal, whether the singularity $k_{z}=1$ in Eq. (14) is passed above or below for a given $\xi$ depends on the sign of $\xi \sqrt{a}+\nu$. Since the main contribution to the integral comes from $|\xi| \sim 1$, it is clear that for $\sqrt{a} \ll \nu$ we essentially repeat the standard derivation of the stopping force for a uniform motion (in which the singularity is passed below). Mathematically, this corresponds to taking the limit $a \rightarrow 0$ first, then $\nu \rightarrow 0$. In this case, we 




FIG. 1: The modified contour to calculate the integral over $\eta$ in Eq. (11). The long vertical lines at the left and right are located at infinitely large $\operatorname{Re} \eta$. The oblique line is at 45 degrees to the horizontal axis. The circle has an infinitely small radius and is centered at the singularity $\eta=\left(-1+k_{z}-\right.$ $i \nu) / \sqrt{k_{z} a}$ if this point lies above the oblique line (otherwise, the circle is not included).

get $F^{\text {pole }}=0$ and $F^{\text {line }}=(1 / 2) \ln \left(k_{\max }^{2}+1\right) \simeq \ln k_{\max }$ (for $k_{\max } \gg 1$, which is the condition to employ the linear perturbation approximation), which is the Bohr stopping force. Furthermore, by using Eqs. (13) and (14), it is easy to show that for $\sqrt{a} \ll \nu$ the correction due to a finite $a$ is small as compared to the correction due to a finite $\nu$.

So let us focus on the limit $\nu \rightarrow 0$ for a finite $a$, which implies the opposite regime, $\sqrt{a} \gg \nu$. To do so, it is sufficient to simply set $\nu=0$ in Eqs. (13) and (14) because a finite $a$ already removes the singularity in Eq. (14); note that we have already used that $\nu>0$ by including the contribution from the pole to Eq. (12).

To calculate $F^{\text {pole }}$, we first make the substitution $\beta=$ $\left(1-k_{z}\right)^{2} /\left(a k_{z}\right)$, where $\beta$ is a new integration variable, so the exponent in Eq. (13) becomes $\exp (i \beta / 2)$. To improve the convergence, we change the integration over $\beta$ from the real to imaginary axis, with the new integration limits being 0 and $+i \infty$. (Again, the result remains the same according to Cauchy's theorem.) Then we expand the integrand in powers of $\sqrt{a}$ and integrate the resulting terms over $\beta$, which yields

$$
\begin{array}{r}
F^{\text {pole }}=\frac{1}{2} \ln \left(k_{\max }^{2}+1\right) \\
+\frac{1}{2} \sqrt{\frac{a}{\pi}} \int_{0}^{k_{\max }} \frac{\left(1-3 k_{\perp}^{2}\right) k_{\perp}}{\left(k_{\perp}^{2}+1\right)^{2}} d k_{\perp}+o(a)
\end{array}
$$

for $a \rightarrow 0$. Note that the term $\propto a$ turns out to be zero. Also note that in the zeroth order, $F^{\text {pole }}$ is the Bohr stopping force (again, for $k_{\max } \gg 1$ ), which is in contrast to the case where the limit $a \rightarrow 0$ is taken before the limit $\nu \rightarrow 0$.

The calculation of $F^{\text {line }}$ in the zeroth order is rather straightforward and yields zero, while in order to calculate the $\sqrt{a}$-term for $F^{\text {line }}$, we differentiate the integrand in Eq. (14) over $\sqrt{a}$ and integrate it over $\xi$ and $\mathbf{k}$ in the limit $a \rightarrow 0$. We consider the integral over $k_{z}$ first and divide it into two parts, one from the vicinity of $k_{z}=1$ and the other one from the rest of the integration interval over $k_{z}$. The latter part obviously cancels out when subsequently integrated over $\xi$, while the former one is easily found by evaluating the corresponding residue. The subsequent integration over $\xi$ is straightforward. The resulting $\sqrt{a}$-term turns out to be minus the $\sqrt{a}$-term in Eq. (15), so they cancel out.

To calculate the next expansion term for $F^{\text {line }}$, we differentiate the integrand in Eq. (14) over $\sqrt{a}$ twice and integrate it over $\xi$ and $\mathbf{k}$ in the limit $a \rightarrow 0$. Since the limit $a \rightarrow 0$ is considered, after the differentiation we can replace $\xi(1+i) \sqrt{k_{z} a}$ in the denominator by $i \varepsilon \xi /|\xi|$, where $\varepsilon$ is an infinitesimal positive number (independent of $k_{z}$ ). We consider the integration over $k_{z}$ first and expand the integration interval from $0<k_{z}<k_{\max }$ to $-\infty<k_{z}<\infty$ as the integral converges well at large $k_{z}$ and the integrand is an even function of $k_{z}$. This modification allows us to integrate over $k_{z}$ by using the residue theorem and Jordan's lemma. The subsequent integration over $\xi$ and $k_{\perp}$ is straightforward - again, since the integral over $k_{\perp}$ converges well at large $k_{\perp}$, we replace the upper limit $k_{\max }$ by $\infty$. The result for the expansion term turns out to be $-\pi a / 2$.

By summing $F^{\text {pole }}$ and $F^{\text {line }}$ and replacing $(1 / 2) \ln \left(k_{\max }^{2}+1\right)$ by $\ln k_{\max }$, we can write

$$
F=F_{0}+\delta F
$$

where, in the dimensional units,

$$
F_{0}=\frac{q^{2} \omega_{\mathrm{pe}}^{2}}{4 \pi \varepsilon_{0} v_{z}^{2}} \ln \left(\frac{k_{\max }\left|v_{z}\right|}{\omega_{\mathrm{pe}}}\right)
$$

is the Bohr stopping force and

$$
\delta F=-\frac{q^{2} \omega_{\mathrm{pe}} a}{8 \varepsilon_{0}\left|v_{z}\right|^{3}}
$$

is the deceleration correction, where we have omitted the higher-order terms with respect to $a$.

Equation (18) is the principal result of this paper. The derived correction is negative, which means that the stopping force is reduced as a result of the deceleration. We also note that the correction does not contain the Coulomb logarithm.

\section{DISCUSSION AND CONCLUSIONS}

To estimate whether and when the deceleration correction can be significant, let us use that the deceleration is caused by the stopping force itself. We assume that the projectile is an ion of charge $Z e$ and mass $A m_{\mathrm{p}}$ and moves with a velocity $v \gtrsim 2 v_{\mathrm{e}}$, the range where the Bohr expression for the stopping force is applicable [1]. Here, $m_{\mathrm{p}}$ is the proton mass, $v_{\mathrm{e}}=\sqrt{k_{\mathrm{B}} T_{\mathrm{e}} / m_{\mathrm{e}}}$ is the electron thermal velocity, $T_{\mathrm{e}}$ is the electron temperature, and $k_{\mathrm{B}}$ is the Boltzmann constant. The deceleration is then taken to be $a=F_{0} /\left(A m_{\mathrm{p}}\right)$. 
First of all, we analyze when the condition $\sqrt{a} \gg \nu$ (in the dimensionless units) holds, i.e., when the deceleration dominates over the damping, as assumed in the derivation of Eqs. (16)-(18). In the dimensional units, this condition reads

$$
\frac{a \omega_{\mathrm{pe}}}{v} \gg \nu^{2}
$$

We substitute one half of the effective electron-ion Coulomb collision frequency for $\nu$ (see Ref. [14]),

$$
\nu=\frac{1}{6(2 \pi)^{3 / 2}} \frac{v_{\mathrm{e}} n e^{4}}{\left(\varepsilon_{0} k_{\mathrm{B}} T_{\mathrm{e}}\right)^{2}} \ln \Lambda_{\mathrm{e}},
$$

where the ions are assumed to be singly ionized, $n$ is the plasma number density,

$$
\Lambda_{\mathrm{e}}=\frac{4 \pi \varepsilon_{0} k_{\mathrm{B}} T_{\mathrm{e}} v_{\mathrm{e}}}{\omega_{\mathrm{pe}} e^{2}}
$$

and $\omega_{\text {pe }}=\left[n e^{2} /\left(\varepsilon_{0} m_{\mathrm{e}}\right)\right]^{1 / 2}$. This allows us to rewrite Eq. (19) as

$$
\frac{1}{\Gamma_{\mathrm{e}}^{3 / 2}} \frac{Z^{2}}{A}\left(\frac{v_{\mathrm{e}}}{v}\right)^{3} \frac{\ln \Lambda_{q}}{\ln ^{2} \Lambda_{\mathrm{e}}} \gg 10^{2},
$$

where the factor $10^{2}$ includes all numerical coefficients as well as the proton-electron mass ratio,

$$
\Gamma_{\mathrm{e}}=\frac{e^{2} n^{1 / 3}}{4 \pi \varepsilon_{0} k_{\mathrm{B}} T_{\mathrm{e}}}
$$

is the electron coupling parameter, and $\Lambda_{q}$ is the argument of the logarithm in Eq. (17). Clearly, the condition (22) is often satisfied - the electrons only need to be sufficiently weakly coupled for that.

The ratio of the deceleration correction (18) to the Bohr stopping force (17) can be written as

$$
\frac{|\delta F|}{F_{0}} \simeq 3 \times 10^{-3} \Gamma_{\mathrm{e}}^{3 / 2} \frac{Z^{2}}{A}\left(\frac{v_{\mathrm{e}}}{v}\right)^{3},
$$

where, again, the factor $3 \times 10^{-3}$ includes all numerical coefficients and the electron-proton mass ratio. We see that there is no way of making this correction comparable to the Bohr stopping force as long as the electrons are weakly coupled [as required by Eq. (22)] and $v \gtrsim 2 v_{\mathrm{e}}$ (as assumed above).

Let us now consider the regime $2 v_{\mathrm{i}} \lesssim v \ll v_{\mathrm{e}}$, where $v_{\mathrm{i}}=\sqrt{k_{\mathrm{B}} T_{\mathrm{i}} / m_{\mathrm{i}}}$ is the ion thermal velocity, $T_{\mathrm{i}}$ is the ion temperature, and $m_{\mathrm{i}}$ is the ion mass. In this case, we can still use Eqs. (16)-(18), but we must replace the quantities characterizing the electron component by those for the ion component. Then the ratio of the deceleration correction to the Bohr stopping force, which is now due to the ions, is

where

$$
\frac{|\delta F|}{F_{0}}=\left(\pi \Gamma_{\mathrm{i}}\right)^{3 / 2} \frac{Z^{2}}{A}\left(\frac{v_{\mathrm{i}}}{v}\right)^{3},
$$

$$
\Gamma_{\mathrm{i}}=\frac{e^{2} n^{1 / 3}}{4 \pi \varepsilon_{0} k_{\mathrm{B}} T_{\mathrm{i}}}
$$

is the ion coupling parameter. [Note that the restriction (22) is inapplicable here as it was derived for electrons; for ions there is no collisional background unless the plasma is partially ionized.] Equation (25) shows that in the regime considered, the deceleration effect can be highly significant. For instance, for $v=2 v_{\mathrm{i}}$ (which is approximately the location of the peak of the stopping force [1]), an $\mathrm{S}^{16+}$-projectile (as, e.g., in the experiments of Ref. [15]), a hydrogen plasma, and $\Gamma_{\mathrm{i}}=0.5$, Eq. (25) yields $\delta F / F_{0} \simeq 2$, meaning that the peak of the stopping force should be greatly reduced as a result of the deceleration effect. Note that the applicability condition of the linear perturbation approximation, which can be written as

$$
\frac{Z e^{2} \omega_{\mathrm{pi}}}{4 \pi \varepsilon_{0} m_{\mathrm{i}} v^{3}}=2 \sqrt{\pi} \Gamma_{\mathrm{i}}^{3 / 2} Z\left(\frac{v_{\mathrm{i}}}{v}\right)^{3} \ll 1
$$

where $\omega_{\mathrm{pi}}=\left[n e^{2} /\left(\varepsilon_{0} m_{\mathrm{i}}\right)\right]^{1 / 2}$ is the ion plasma frequency, is violated for these parameter values as the left-hand side of the inequality (27) is $\simeq 2.5$, but it seems highly unrealistic to expect that nonlinear effects can make the deceleration correction insignificant. For a $\mathrm{He}^{2+}$-projectile, the left-hand side of the inequality (27) is $\simeq 0.3$, while $\delta F / F_{0} \simeq 0.24$, which is still a noticeable correction.

To conclude, we have rigorously addressed the effect of the projectile deceleration on the stopping force. We have provided a general expression that allows investigating this effect for any specified dielectric function [Eq. (7)]. For a cold plasma, we have derived a simple expression for the correction to the stopping force [Eq. (18)]. Assuming that the projectile is an ion, we have found that this correction is small when the stopping force is due to the plasma electrons, but can be significant when the stopping force is due to the ions.

\section{Acknowledgments}

The authors thank Mikhail Pustylnik for useful discussions. The work received funding from the European Research Council under the European Union's Seventh Framework Programme (FP7/2007-2013)/ERC Grant agreement 267499.
[1] T. Peter and J. Meyer-Ter-Vehn, Phys. Rev. A 43, 1998 (1991).
[2] D. H. H. Hoffmann, R. Bock, A. Y. Faenov, U. Funk, 
M. Geissel, U. Neuner, T. A. Pikuz, F. Rosmej, M. Roth, W. Süß, et al., Nucl. Instrum. Meth. B 161, 9 (2000).

[3] S. Kawata, K. Horioka, M. Murakami, Y. Oguri, J. Hasegawa, K. Takayama, H. Yoneda, K. Miyazawa, T. Someya, A. I. Ogoyski, et al., Nucl. Instrum. Meth. A 577, 21 (2007).

[4] M. Lampe, G. Joyce, G. Ganguli, and V. Gavrishchaka, Phys. Plasmas 7, 3851 (2000).

[5] S. A. Khrapak, A. V. Ivlev, G. E. Morfill, and H. M. Thomas, Phys. Rev. E 66, 046414 (2002).

[6] V. Yaroshenko, S. Ratynskaia, S. Khrapak, M. H. Thoma, M. Kretschmer, H. Höfner, G. E. Morfill, A. Zobnin, A. Usachev, O. Petrov, et al., Phys. Plasmas 12, 093503 (2005).

[7] O. Ishihara, J. Phys. D: Appl. Phys. 40, 121 (2007).

[8] W. J. Miloch, M. Kroll, and D. Block, Phys. Plasmas 17,
103703 (2010).

[9] A. V. Ivlev, S. K. Zhdanov, S. A. Khrapak, and G. E. Morfill, Phys. Rev. E 71, 016405 (2005).

[10] N. Bohr, Philos. Mag. 30, 581 (1915).

[11] T. Peter, J. Plasma Phys. 44, 269 (1990).

[12] R. Kompaneets, S. V. Vladimirov, A. V. Ivlev, and G. Morfill, New J. Phys. 10, 063018 (2008).

[13] E. M. Lifshitz and L. P. Pitaevskii, Physical Kinetics (Pergamon, Oxford, 1981).

[14] A. F. Alexandrov, L. S. Bogdankevich, and A. A. Rukhadze, Principles of Plasma Electrodynamics (Springer, Berlin, 1984).

[15] H.-D. Betz, R. Höppler, R. Schramm, and W. Oswald, Nucl. Instrum. Meth. B 33, 185 (1988). 